\title{
BRAF V600E Mutation Independently Predicts Central Compartment Lymph Node Metastasis in Patients with Papillary Thyroid Cancer
}

\author{
Martha A. Zeiger, MD, FACS and Eric B. Schneider, PhD \\ Division of Endocrine Surgery, Department of Surgery, Johns Hopkins University School of Medicine, Baltimore, MD
}

Howell et al. examine a very timely question regarding the utility of measuring BRAF mutation status in papillary thyroid cancer (PTC) specimens to determine if it can be used to predict the presence or absence of lymph node metastases. The premise is that its measurement could potentially guide the surgical management of patients with PTC; i.e., to determine whether a prophylactic central lymph node dissection (CLND) should be performed. This is a very well-written study that nicely captures the common clinical dilemma faced by endocrine surgeons and also is comprehensive in the interpretation of its results.

It is generally accepted that patients with PTC who have clinically detectable lymph node metastases either on physical examination or by ultrasound should undergo a lymph node dissection. Whether to perform a prophylactic CLND for PTC remains debatable; some authors claim that there is no advantage in terms of decreased local recurrence and improved survival, whereas others claim advantages regarding both. ${ }^{1}$ Because of this controversy, some authors have proposed that $B R A F$ may confer a more aggressive phenotype and its measurement could then help to guide the surgeon in terms of whether or not to perform a prophylactic CLND. Conversely, other authors have documented no correlation with BRAF mutation and aggressive features of PTC, including the case in point, namely, the presence of lymph node metastases..$^{2-4}$ The literature is replete with studies that include only patients who had therapeutic and not routine prophylactic CLND, patients who had lymph nodes removed only incidentally, or patients who had no lymph nodes removed; none of

(C) Society of Surgical Oncology 2012

Published Online: 1 September 2012

M. A. Zeiger, MD, FACS

e-mail: mzeiger@jhmi.edu these groups of patients would therefore be evaluable in terms of determining whether $B R A F$ status is truly associated with CLN metastases. Two studies have included only patients who have undergone routine CLND and both found that $B R A F$ mutation predicted metastatic disease only in patients who had a PTC that was less than $1 \mathrm{~cm}$ in size; there was no association for larger tumors. ${ }^{5,6}$

Howell et al. initially identified 274 patients who were operated on for PTC at the University of Pittsburgh in 2010 and from this group studied $156(57 \%)$ patients who had both lymph nodes resected and $B R A F$ testing. In their study, therapeutic CLND was performed for patients who had evidence of metastatic disease either on ultrasonography or at the time of surgery. A prophylactic CLND was performed for patients who had a diagnosis of PTC or a positive BRAF mutation. If PTC was not diagnosed preoperatively, the lymph nodes were assessed with visual and tactile inspection and patients "had nonanatomic resection of immediate perithyroidal central compartment lymph nodes if no suspicious lymphadenopathy was identified." Incidentally, we know from others' work that intraoperative assessment of lymph nodes is generally unreliable. ${ }^{7-9}$ In the study by Howell et al. 29 (19\%) had a therapeutic CLND, $85(54 \%)$ had a prophylactic CLND, and 42 $(27 \%)$ had incidental central lymph nodes resected. In the entire group of $156,37 \%$ had evidence of metastatic disease and $46 \%$ were $B R A F$ mutation-positive. They found that $B R A F$ mutation was associated with lymph node metastases both in univariate analysis and multivariable logistic regression. The sensitivity was noted to be $62 \%$ and the specificity was $63 \%$; the positive and negative predictive values were 50 and $74 \%$, respectively.

The study by Howell et al. includes patients who have undergone some sort of lymph node removal. It excludes, however, more than $40 \%$, because they did not have a 
CLND. Specifically, 118 of 274 (43\%) patients were excluded: $51(19 \%)$ because no molecular testing was performed, and $67(24 \%)$ because they did not have a true CLND. Furthermore, of the final 156 patients who were included, $42(27 \%)$ did not have a true CLND. By excluding a high percentage of patients for various reasons and including a large percentage who did not have a true CLND, the concern is that the authors may have introduced significant bias up front, both seen and unseen, into the study design. This study does an excellent job of examining the relationship between $B R A F$ mutation and lymph node metastases in various subsets of their PTC patients who underwent therapeutic or prophylactic CLND. It does not, however, provide information on the relationship between $B R A F$ and lymph node metastases in the $43 \%$ of their patients with histologic PTC who did not meet their clinical criteria for perioperative CLND.

The question that needs to be answered includes the following: does $B R A F$ predict the presence of metastases in PTC patients when metastatic disease is not suspected preoperatively, i.e., when preoperative ultrasound is negative for metastatic disease? The authors nicely evaluated a subset of 127 patients without preoperative evidence of metastatic disease who underwent prophylactic or incidental CLND and found that BRAF did indeed predict metastases with a sensitivity of $59 \%$ and a specificity of $63 \%$. However, these 127 individuals were not a random sample of PTC patients, because one of the criteria for prophylactic CLND was the presence of $B R A F$ mutation in preoperative molecular testing. Using $B R A F$ status as a criterion for prophylactic CLND may skew the observations, because the subset of study patients undergoing CLND may be disproportionately more likely to carry the $B R A F$ mutation compared with the general population of PTC patients.

The clinically relevant information that can be gleaned from this study includes the following: overall, 36 of 72 (50\%) patients who were $B R A F$-positive had lymph node metastases, whereas 22 of $84(26 \%)$ patients who were $B R A F$-negative had lymph node metastases; within the prophylactic group, 19 of 54 (35\%) of patients who were $B R A F$-positive had lymph node metastases, whereas only 13 of 73 (18\%) of patients who were $B R A F$-negative had lymph node metastases. In light of possible biases introduced by including $B R A F$ status as a criterion for prophylactic CLND, are the reported findings of this study sufficient to guide the future clinical management of patients with PTC? Even with questions related to selection bias set aside, should the surgeon perform a prophylactic CLND if the patient is likely to harbor metastatic disease $50 \%$ of the time if they are $B R A F$-positive compared with $26 \%$ of the time if they are $B R A F$-negative, or, looking at only the prophylactic group, should the surgeon perform a prophylactic CLND if the patient is likely to harbor metastatic disease $35 \%$ of the time if they are $B R A F$-positive compared with $18 \%$ of the time if they are $B R A F$-negative? These differences would be unlikely to influence surgical decision making in a meaningful way.

Overall, this very well-written manuscript addresses an important clinical question and contributes substantially to the foundation of data supporting the need for further studies. For several reasons catalogued above, caution must be exercised when attempting to extrapolate the results of this excellent study to a broader population. Future studies should include evaluation of consecutive patients who present with a diagnosis of PTC and who undergo true CLND. It is only through prospective study that the effect of $B R A F$ mutation on the risk of developing lymph node metastases can be elucidated fully. Interpretation of the results of future studies should include not only statistical analysis but also its practical application; in other words, what sensitivity, specificity, and positive and negative predictive values would truly influence and impact patient care? Going forward, should $B R A F$ status determine the surgical treatment of PTC, or will $B R A F$ status ultimately contribute to an evidence-based algorithm that will guide best practices in the surgical treatment of PTC? Only time and carefully designed prospective studies will tell.

\section{REFERENCES}

1. Mazzaferri EL, Doherty GM, Steward DL. The pros and cons of prophylactic central compartment lymph node dissection for papillary thyroid carcinoma. Thyroid. 2009;19(7):683-9.

2. Fugazzola L, Mannavola D, Cirello V, et al. BRAF mutations in an Italian cohort of thyroid cancers. Clin Endocrinol. 2004;61(2): 239-43.

3. Ito $\mathrm{Y}$, Yoshida $\mathrm{H}$, Maruo $\mathrm{R}$, et al. BRAF mutation in papillary thyroid carcinoma in a Japanese population: its lack of correlation with high-risk clinicopathological features and disease-free survival of patients. Endocr J. 2009;56(1):89-97.

4. Xing M, Clark D, Guan H, et al. BRAF mutation testing of thyroid fine-needle aspiration biopsy specimens for preoperative risk stratification in papillary thyroid cancer. J Clin Oncol. 2009; 27(18):2977-82.

5. Lee X, Gao M, Ji Y, et al. Analysis of differential BRAF(V600E) mutational status in high aggressive papillary thyroid microcarcinoma. Ann Surg Oncol. 2009;16(2):240-5.

6. So YK, Son YI, Park JY, et al. Preoperative BRAF mutation has different predictive values for lymph node metastasis according to tumor size. Otolaryngol Head Neck Surg. 2011;145(3):422-7.

7. Moley JF, DeBenedetti MK. Patterns of nodal metastases in palpable medullary thyroid carcinoma: recommendations for extent of node dissection. Ann Surg. 1999;229(6):880-7; discussion $887-888$.

8. Teixeira G, Teixeira T, Gubert F, et al. The incidence of central neck micrometastatic disease in patients with papillary thyroid cancer staged preoperatively and intraoperatively as N0. Surgery. 2011;150(6):1161-7.

9. Hwang HS, Orloff LA. Efficacy of preoperative neck ultrasound in the detection of cervical lymph node metastasis from thyroid cancer. Laryngoscope. 2011;121(3):487-91. 